\title{
Effect of lanreotide on postoperative health outcomes in patients with acromegaly
}

Treatment with the somatostatin analog lanreotide before transsphenoidal surgery improves surgical cure rates in patients with acromegaly as a result of growth-hormone-secreting pituitary macroadenomas, but has no effect on surgical complications or duration of hospitalization, say researchers of a study published in the European Journal of Endocrinology.

\section{4 ...preoperative treatment} with lanreotide could improve surgical cure rates of patients with acromegaly... 77

Transsphenoidal surgery for the selective removal of pituitary adenomas is currently the first-line treatment for most patients with acromegaly. Success rates for the surgery of macroadenomas, however, are lower than those for microadenomas. Lanreotide, a synthetic somatostatin analog that inhibits the release of growth hormone, TSH, insulin and glucagon, could potentially reduce the size of growth-hormone-secreting pituitary adenomas.

Previous small, retrospective studies have addressed preoperative treatment with somatostatin analogs and subsequent surgical cure rates, but the results were conflicting. Mao et al., therefore, conducted a prospective, randomized study to investigate whether preoperative treatment with lanreotide could improve surgical cure rates of patients with acromegaly as a result of a macroadenoma.

The researchers randomly assigned 98 patients to either a 4 -month preoperative treatment with lanreotide (starting dose $30 \mathrm{mg}$ every 2 weeks, titrated to a weekly dose of $30 \mathrm{mg}$ after 8 weeks if the mean growth hormone level was $>2.5 \mu \mathrm{g} / \mathrm{l}$ ) or to transsphenoidal surgery alone. Surgical cure rates were determined 4 months after the operation primarily by measuring fasting levels of insulin-like growth factor 1 .

Surgical cure was established in 24 of 49 patients pretreated with lanreotide compared with 9 of 49 patients who were treated with surgery alone. The occurrence of surgical morbidity was similar between groups, as was the postoperative hospital stay $(4.5 \pm 1.6$ days in patients treated with lanreotide versus $4.8 \pm 1.9$ days in patients treated with surgery alone).

\section{Linda Koch}

Original article Mao, Z. G. et al. Preoperative lanreotide treatment in acromegalic patients with macroadenomas increases short-term postoperative cure rates: a prospective, randomized trial. Eur. J. Endocrinol. doi:10.1530/eje-09-090 